\title{
Diurnal variation of dust and gas production in comet 67P/Churyumov-Gerasimenko at the inbound equinox as seen by OSIRIS and VIRTIS-M on board Rosetta
}

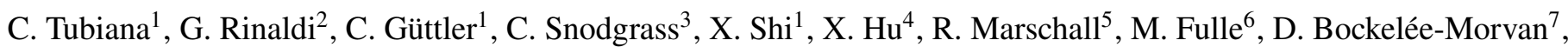
G. Naletto ${ }^{25}$, F. Capaccioni ${ }^{2}$, H. Sierks ${ }^{1}$, G. Arnold ${ }^{9}$, M. A. Barucci ${ }^{7}$, J.-L. Bertaux ${ }^{16}$, I. Bertini ${ }^{17}$, D. Bodewits ${ }^{11}$, M. T. Capria ${ }^{2}$, M. Ciarniello ${ }^{2}$, G. Cremonese ${ }^{18}$, J. Crovisier ${ }^{7}$, V. Da Deppo ${ }^{19}$, S. Debei ${ }^{20}$, M. De Cecco ${ }^{21}$, J. Deller ${ }^{1}$, M. C. De Sanctis ${ }^{2}$, B. Davidsson ${ }^{15}$, L. Doose ${ }^{10}$, S. Erard ${ }^{7}$, G. Filacchione ${ }^{2}$, U. Fink ${ }^{10}$, M. Formisano ${ }^{2}$, S. Fornasier ${ }^{7}$, P. J. Gutiérrez ${ }^{22}$, W.-H. Ip ${ }^{26,27}$, S. Ivanovski ${ }^{6}$, D. Kappel ${ }^{23,9}$, H. U. Keller ${ }^{24,9}$, L. Kolokolova ${ }^{28}$, D. Koschny ${ }^{14}$, H. Krueger ${ }^{1}$, F. La Forgia ${ }^{17}$, P. L. Lamy ${ }^{12}$, L. M. Lara ${ }^{22}$, M. Lazzarin ${ }^{17}$, A. C. Levasseur-Regourd ${ }^{30}$, Z.-Y. Lin ${ }^{26}$, A. Longobardo ${ }^{2,31}$, J. J. López-Moreno ${ }^{22}$, F. Marzari ${ }^{25}$, A. Migliorini ${ }^{2}$, S. Mottola ${ }^{9}$, R. Rodrigo ${ }^{13,5}$, F. Taylor ${ }^{8}$, I. Toth $^{29}$, and V. Zakharov ${ }^{2}$

(Affiliations can be found after the references)

Received 14 December 2018 / Accepted 6 May 2019

\section{ABSTRACT}

\begin{abstract}
Context. On 27 April 2015, when comet 67P/Churyumov-Gerasimenko was at 1.76 au from the Sun and moving toward perihelion, the OSIRIS and VIRTIS-M instruments on board the Rosetta spacecraft simultaneously observed the evolving dust and gas coma during a complete rotation of the comet.

Aims. We aim to characterize the spatial distribution of dust, $\mathrm{H}_{2} \mathrm{O}$, and $\mathrm{CO}_{2}$ gas in the inner coma. To do this, we performed a quantitative analysis of the release of dust and gas and compared the observed $\mathrm{H}_{2} \mathrm{O}$ production rate with the rate we calculated using a thermophysical model.

Methods. For this study we selected OSIRIS WAC images at $612 \mathrm{~nm}$ (dust) and VIRTIS-M image cubes at $612 \mathrm{~nm}, 2700 \mathrm{~nm}\left(\mathrm{H}_{2} \mathrm{O}\right.$ emission band), and $4200 \mathrm{~nm}\left(\mathrm{CO}_{2}\right.$ emission band). We measured the average signal in a circular annulus to study the spatial variation around the comet, and in a sector of the annulus to study temporal variation in the sunward direction with comet rotation, both at a fixed distance of $3.1 \mathrm{~km}$ from the comet center.

Results. The spatial correlation between dust and water, both coming from the sunlit side of the comet, shows that water is the main driver of dust activity in this time period. The spatial distribution of $\mathrm{CO}_{2}$ is not correlated with water and dust. There is no strong temporal correlation between the dust brightness and water production rate as the comet rotates. The dust brightness shows a peak at $0^{\circ}$ subsolar longitude, which is not pronounced in the water production. At the same epoch, there is also a maximum in $\mathrm{CO}_{2}$ production. An excess of measured water production with respect to the value calculated using a simple thermophysical model is observed when the head lobe and regions of the southern hemisphere with strong seasonal variations are illuminated (subsolar longitude $270^{\circ}-50^{\circ}$ ). A drastic decrease in dust production when the water production (both measured and from the model) displays a maximum occurs when typical northern consolidated regions are illuminated and the southern hemisphere regions with strong seasonal variations are instead in shadow (subsolar longitude $50^{\circ}-90^{\circ}$ ). Possible explanations of these observations are presented and discussed.
\end{abstract}

Key words. comets: general - comets: individual: 67P/Churyumov-Gerasimenko - methods: data analysis

\section{Introduction}

After a ten-year journey and 30 months of deep-space hibernation, the ESA Rosetta spacecraft woke up on 20 January 2014. Rosetta had the unique opportunity to stay in the vicinity of comet 67P/Churyumov-Gerasimenko (hereafter 67P) for $2.5 \mathrm{yr}$ and to observe how the comet evolved while moving along its orbit.

One of the main goals of the Rosetta mission was to understand cometary activity, that is, the physical processes that generate the dust and gas coma from the nucleus. While the broad picture of the Whipple model, that is, that ices in the nucleus sublimate when heated by the Sun and the resulting gas outflow lifts dust (Whipple 1950), has been confirmed by observations, details of the processes involved remain the subject of debate. Observations of dust jets have been traced to certain areas of the surface (Vincent et al. 2016), and various models have been developed to trace gas and dust flow in the inner coma of 67P, with varying degrees of complexity (e.g., Fougere et al. 2016; Kramer \& Noack 2016; Kramer et al. 2017; Zakharov et al. 2018), but the models do not yet uniquely identify the surface features that are responsible for activity. Many of the models show that the bulk activity can be explained by more or less homogeneous activity from all illuminated surface facets (Keller et al. 2015) and that jets in the inner coma are controlled more by the complex shape of the nucleus than by anything special about their apparent source on the surface (Shi et al. 2018a). There are clear variations in activity with seasonal illumination of the 
comet, however, which appear to be related to the very different morphology of the northern and southern surfaces, and models that explain the early activity seen by Rosetta when 67P was far from the Sun do not reproduce the perihelion behavior (Shi et al. 2018b). Recent models attempt to reproduce the complexities of the changing activity (Attree et al. 2019; Marschall et al. 2019); they also include investigations of the relative contribution of different sublimating ices (water or $\mathrm{CO}_{2}$ ) to the driving activity, that is, how the ices are related to each other (Gasc et al. 2017) and to dust release.

The aim of this work is to take advantage of the capabilities of two instruments on Rosetta to analyze the dust and gas coma behavior in the pre-perihelion phase, when the comet was at heliocentric distances of 1.76 au and close to the equinox between the changing seasons. This allows us to investigate the differences in observed dust and gas distributions and their longitudinal variations simultaneously over a full rotation of the nucleus. We investigate both the spatial variation in dust and gas around the comet and how it varies with time as different areas are illuminated throughout the cometary day.

\section{Instruments, datasets, and methods}

\subsection{Instruments}

The Optical, Spectroscopic, and Infrared Remote Imaging System (OSIRIS) and the Visible InfraRed Thermal Imaging Spectrometer (VIRTIS) were 2 of the 12 scientific instruments on board the Rosetta orbiter (Glassmeier et al. 2007). OSIRIS (Keller et al. 2007) was the scientific camera system. It comprised a Narrow Angle Camera (NAC) and a Wide Angle Camera (WAC) with fields of view (FOV) of $2.20^{\circ} \times 2.22^{\circ}$ and $11.35^{\circ} \times 12.11^{\circ}$, respectively. Both cameras used a $2048 \times$ 2048 pixel backside-illuminated CCD detector with a UV optimized antireflection coating. The CCDs were equipped with lateral antiblooming that allowed overexposure of the nucleus without creating saturation artifacts, enabling the study of details in the faint coma structures next to the illuminated limb. The NAC was equipped with 11 filters covering the wavelength range 250-1000 nm, while the WAC had 14 filters covering the range 240-720 nm (Tubiana et al. 2015).

The VIRTIS spectrometer (Coradini et al. 2007) was composed of two spectral channels: VIRTIS-M and VIRTIS-H. VIRTIS-M was the visible (230-1000 nm, 432 bands) and infrared (1000-5000 nm, 432 bands) imaging spectrometer with a field of view of $3.6^{\circ}$ (along the slit axis) and an instantaneous field of view (IFOV) of $250 \mu \mathrm{rad}$. The instrument acquired hyperspectral cubes by scanning in time the target scene line by line. The duration of the acquisition ( $\Delta t$ in Table 1$)$ is given by the number of lines (including periodic dark current frames) times the internal repetition time, where the repetition time is the time between two consecutive steps that are required to move the internal scan mirror by one IFOV. The integration time ( $t_{\exp }$ in Table 1) set for the VIS and IR channels was lower than the internal repetition time. The maximum $3.6^{\circ} \times 3.6^{\circ} \mathrm{FOV}$ was imaged by repeating acquisition on successive 256 scan mirror steps (lines). From a distance of $100 \mathrm{~km}$, this corresponds to a $6.4 \mathrm{~km} \times$ $6.4 \mathrm{~km}$ swath with a resolution of $25 \mathrm{~m} \mathrm{pix}^{-1}$. As an example, we show in Fig. 1 a VIRTIS-M hyperspectral cube with the line and time axes. VIRTIS-H was the infrared high-spectral resolution point spectrograph operating in the $1900-5000 \mathrm{~nm}$ spectral range with a $\lambda / \Delta \lambda=1300 \div 3000$. The instrument observed in a single IFOV of $580 \mu \mathrm{rad} \times 1740 \mu \mathrm{rad}$, which corresponds to a resolution of $58 \mathrm{~m} \times 174 \mathrm{~m}$ from a $100 \mathrm{~km}$ distance. Because the
VIRTIS-H and VIRTIS-M boresights were coaligned, the point spectrograph was able to acquire the same area several times during the time required for a scan for the imaging channel.

\subsection{Datasets}

Throughout the entire mission, we regularly carried out socalled dust monitoring sequences, which were designed to observe the coma of unresolved dust particles. Typically, these sequences spanned at least $12 \mathrm{~h}$ (i.e., a full comet rotation) with hourly cadence. In the frame of this work, we have analyzed one dust monitoring sequence that was acquired on 27 April $2015^{1}$. At the time of the observation, 67P was at a heliocentric distance of $1.76 \mathrm{au}$, moving toward perihelion, and close to the equinox between the long but cool northern summer and the short intensely illuminated southern summer around perihelion. The Rosetta spacecraft was at a distance between 125 and $142 \mathrm{~km}$ from the comet. The Rosetta $+Z$-axis was pointing to the comet nucleus (IlluminatedPoint pointing), and because of the spacecraft-comet distance, the nucleus was entirely contained in all OSIRIS WAC images and in seven out of nine VIRTIS-M images.

The spacecraft was approximately in a terminator orbit (phase angle $\sim 73^{\circ}-75^{\circ}$ ) so that one side of the comet was illuminated by the Sun and the other side was in darkness. The observational details are summarized in Tables 1 and 2. The dataset of 27 April 2015 is one of the best acquired by VIRTIS-M for this purpose because it covers both the VIS and IR channels and spans more than $12 \mathrm{~h}$. It was one of the final monitoring observations before the failure of the VIRTIS-M cryocooler disabled the IR channel. It was optimized for coma observations and achieved a high signal-to-noise ratio $(\mathrm{S} / \mathrm{N})$. The same dataset was previously analyzed by Rinaldi et al. (2016) and Fink et al. (2016). Rinaldi et al. (2016) focused their analysis on the comparison between the spatial distributions of dust and $\mathrm{H}_{2} \mathrm{O}$ and $\mathrm{CO}_{2}$ gas, radial profiles, and azimuthal distributions to search for any correlation between them. Fink et al. (2016) focused their investigation on the emission intensity of $\mathrm{CO}_{2}$ and $\mathrm{H}_{2} \mathrm{O}$ and provided an explanation for the large observed variations reported in the literature for the $\mathrm{CO}_{2}$-to- $\mathrm{H}_{2} \mathrm{O}$ ratio. In this work we study temporal variation in more detail, and can better study areas of the dust continuum that were heavily affected by stray light in the VIRTIS-M data by combining it with OSIRIS imaging.

\subsubsection{OSIRIS dataset}

The dust monitoring sequence STP053_DUST_MON_006 contained 45 WAC full-frame images. For this study we selected the 15 images acquired with the VIS610 filter $\left(\lambda_{\text {cent }}=612.6 \mathrm{~nm}, \Delta \lambda=\right.$ $9.8 \mathrm{~nm}$ ) and with exposure time optimized for dust coma studies. One image was acquired with the WAC door closed and it was excluded from the analysis. Thus, we used 14 OSIRIS images in total. We used OSIRIS level 3 (CODMAC Level 4) images, which are radiometric calibrated and geometric distortion corrected (for details, see a description of the OSIRIS calibration pipeline in Tubiana et al. 2015). The images, scaled to the same intensity levels, are shown in Fig. 3 (first column).

\subsubsection{VIRTIS-M dataset}

A set of nine image cubes was obtained. The image cubes in the two channels were taken at the same time, with the VIS exposure

\footnotetext{
1 The data are available at the Planetary Science Archive of the European Space Agency under https://www.cosmos.esa.int/web/ psa/rosetta
} 
Table 1. VIRTIS-M observational details.

\begin{tabular}{|c|c|c|c|c|c|c|c|c|c|}
\hline$\#$ & File name & $S_{\text {cube }}$ & $t_{\text {start }}(\mathrm{UTC})$ & $\Delta t(\mathrm{~s})$ & $t_{\exp }(\mathrm{s})$ & $d_{\mathrm{S} / \mathrm{C}}(\mathrm{km})$ & $R\left(\mathrm{mpix}^{-1}\right)$ & $\phi_{ \pm 45^{\circ}}\left(^{\circ}\right)$ & $\phi_{360^{\circ}}\left(^{\circ}\right)$ \\
\hline & \multicolumn{9}{|l|}{ VIRTIS-M-IR } \\
\hline$\overline{1}$ & I1_00388760487.CAL & 256264432 & $13: 02: 43$ & 2778 & 3 & 128 & 32 & 140.0 & 132.9 \\
\hline 2 & I1_00388763546.CAL & 256264432 & $13: 53: 41$ & 2778 & 3 & 130 & 32 & 115.4 & 108.3 \\
\hline 3 & I1_00388766847.CAL & 256264432 & $14: 48: 43$ & 2778 & 3 & 131 & 33 & 88.7 & 81.7 \\
\hline 4 & I1_00388770446.CAL & 256264432 & $15: 48: 41$ & 2778 & 3 & 132 & 33 & 59.7 & 52.8 \\
\hline 5 & I1_00388776027.CAL & 256258432 & $17: 21: 43$ & 5415 & 3 & 134 & 34 & 9.3 & 355.9 \\
\hline 6 & I1_00388781546.CAL & 256258432 & $18: 53: 41$ & 5415 & 3 & 136 & 34 & 324.8 & 311.6 \\
\hline 7 & I1_00388787067.CAL & 256220432 & $20: 25: 43$ & 4635 & 3 & 139 & 35 & 280.2 & 268.4 \\
\hline 8 & I1_00388794147.CAL & 256133432 & $22: 23: 43$ & 1398 & 3 & 141 & 35 & 228.6 & 222.9 \\
\hline \multirow[t]{2}{*}{9} & I1_00388795646.CAL & 256133432 & $22: 48: 41$ & 1398 & 3 & 142 & 35 & 216.6 & - \\
\hline & \multicolumn{9}{|l|}{ VIRTIS-M-VIS } \\
\hline$\overline{10}$ & V1_00388760489.CAL & 256264432 & $13: 02: 45$ & 2778 & 5 & 128 & 32 & 140.0 & 132.9 \\
\hline 11 & V1_00388763549.CAL & 256264432 & $13: 53: 45$ & 2778 & 5 & 130 & 32 & 115.4 & 108.3 \\
\hline 12 & V1_00388766849.CAL & 256264432 & $14: 48: 45$ & 2778 & 5 & 131 & 33 & 88.7 & 81.7 \\
\hline 13 & V1_00388770449.CAL & 256264432 & $15: 48: 45$ & 2778 & 5 & 132 & 33 & 59.7 & 52.8 \\
\hline 14 & V1_00388776036.CAL & 256258432 & $17: 21: 52$ & 5415 & 5 & 134 & 34 & 9.3 & 355.9 \\
\hline 15 & V1_00388781556.CAL & 256258432 & $18: 53: 52$ & 5415 & 5 & 136 & 34 & 324.8 & 311.6 \\
\hline 16 & V1_00388787076.CAL & 256220432 & $20: 25: 52$ & 4635 & 5 & 139 & 35 & 280.2 & 268.4 \\
\hline 17 & V1_00388794149.CAL & 256133432 & $22: 23: 45$ & 1398 & 5 & 141 & 35 & 228.6 & 222.9 \\
\hline 18 & V1_00388795649.CAL & 256133432 & $22: 48: 45$ & 1398 & 5 & 142 & 35 & 216.6 & - \\
\hline
\end{tabular}

Notes. Column 1: assigned number for each image cube; Col. 2: observation file names; Col. 3: image cube dimension in number of samples (256 fixed pixel number), number of scan lines and spectral bands (432 for each channel); Col. 4: start time of each image cube [UTC]; Col. 5: total duration for each image cube; Col. 6: exposure time for each line; Col. 7: S/C distance from the comet center; Col. 8: pixel dimension at the distance of each observation; Col. 9: subsolar longitude of each observation at the time of the middle of the $\pm 45^{\circ}$ sector (see Fig. 1 and Sect. 2.3); Col. 10: subsolar longitude of each observation at the time of the middle of the annulus (see Fig. 1 and Sect. 2.3).

Table 2. OSIRIS observational details.

\begin{tabular}{|c|c|c|c|c|c|c|}
\hline \# & File name & $t_{\text {start }}(\mathrm{UTC})$ & $t_{\exp }(\mathrm{s})$ & $d_{\mathrm{S} / \mathrm{C}}(\mathrm{km})$ & $R\left(\mathrm{~m} \mathrm{pix}^{-1}\right)$ & $\phi\left(^{\circ}\right)$ \\
\hline $\mathrm{a}$ & WAC_2015-04-27T09.24.16.464Z_ID30_1397549300_F18.IMG & $09: 25: 32$ & 7.8 & 125 & 12.4 & 249.7 \\
\hline $\mathrm{b}$ & WAC_2015-04-27T10.24.16.524Z_ID30_1397549400_F18.IMG & $10: 25: 32$ & 7.8 & 126 & 12.6 & 220.7 \\
\hline $\mathrm{c}$ & WAC_2015-04-27T11.25.18.671Z_ID30_1397549500_F18.IMG & $11: 26: 34$ & 7.8 & 127 & 12.7 & 191.3 \\
\hline $\mathrm{d}$ & WAC_2015-04-27T13.04.01.065Z_ID30_1397549200_F18.IMG & $13: 05: 16$ & 7.8 & 129 & 12.9 & 143.6 \\
\hline $\mathrm{e}$ & WAC_2015-04-27T13.59.00.784Z_ID30_1397549300_F18.IMG & 14:00:16 & 7.8 & 130 & 13.0 & 117.1 \\
\hline $\mathrm{f}$ & WAC_2015-04-27T14.59.00.701Z_ID30_1397549400_F18.IMG & 15:00:16 & 7.8 & 132 & 13.1 & 88.1 \\
\hline $\mathrm{g}$ & WAC_2015-04-27T15.59.00.505Z_ID30_1397549500_F18.IMG & $16: 00: 16$ & 7.8 & 133 & 13.2 & 59.2 \\
\hline $\mathrm{h}$ & WAC_2015-04-27T16.28.59.503Z_ID30_1397549400_F18.IMG & $16: 30: 15$ & 7.8 & 134 & 13.3 & 44.7 \\
\hline $\mathrm{i}$ & WAC_2015-04-27T17.29.57.713Z_ID30_1397549100_F18.IMG & $17: 31: 13$ & 7.8 & 135 & 13.4 & 15.3 \\
\hline $\mathrm{j}$ & WAC_2015-04-27T18.17.57.683Z_ID30_1397549200_F18.IMG & $18: 19: 13$ & 7.8 & 136 & 13.5 & 352.1 \\
\hline $\mathrm{k}$ & WAC_2015-04-27T19.17.57.677Z_ID30_1397549300_F18.IMG & $19: 19: 13$ & 7.8 & 137 & 13.7 & 323.2 \\
\hline 1 & WAC_2015-04-27T20.17.57.647Z_ID30_1397549400_F18.IMG & $20: 19: 13$ & 7.8 & 139 & 13.8 & 294.2 \\
\hline $\mathrm{m}$ & WAC_2015-04-27T20.42.57.691Z_ID30_1397549300_F18.IMG & $20: 44: 13$ & 7.8 & 139 & 13.9 & 282.2 \\
\hline $\mathrm{n}$ & WAC_2015-04-27T22.33.00.788Z_ID30_1397549400_F18.IMG & $22: 34: 16$ & 7.8 & 142 & 14.1 & 229.1 \\
\hline
\end{tabular}

Notes. Column 1: assigned letter for each image; Col. 2: observation file names; Col. 3: start time of each image cube [UTC]; Col. 4: exposure time; Col. 5: S/C distance from the comet center; Col. 6: pixel dimension at the distance of each observation; Col. 7: subsolar longitude of each observation.

sequences starting about $2-4 \mathrm{~s}$ after the IR sequences. The first seven cubes have a FOV of about $9.0 \mathrm{~km} \times 7.7 \mathrm{~km}$ (at the nucleus center distance), and the position of the cometary nucleus is roughly in the center of the image. The last two cubes have a FOV of about $9.0 \mathrm{~km} \times 4 \mathrm{~km}$, and the nucleus is only partially contained in the frame. Figure 3 shows the spatial distribution of dust $(612 \mathrm{~nm})$, water vapor $(2700 \mathrm{~nm})$, and carbon dioxide $(4200 \mathrm{~nm})$. Each map is a composite image where the comet nucleus, taken at $4200 \mathrm{~nm}$, is superimposed on the maps of the dust continuum at $612 \mathrm{~nm}$ (second column), the water vapor (third column), and the $\mathrm{CO}_{2}$ band intensities (fourth column). When the bright nucleus partially illuminates the instrument slit, a sizable fraction of the incoming photons is spread into the adjacent coma pixels. The measured dust continuum is contaminated by stray light caused by the nucleus and cannot be used. The data cubes used for the analysis were calibrated using the 

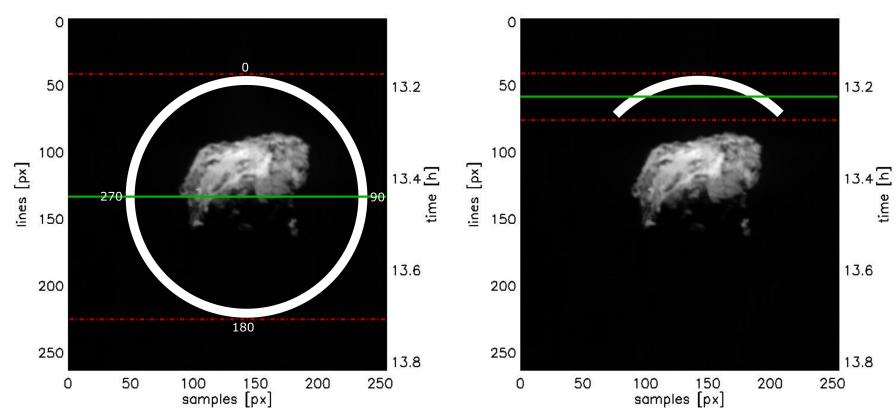

Fig. 1. Example of masks used for aperture photometry measurements: the $\pm 45^{\circ}$ sector (right panel) and the annulus (left panel) superimposed on a VIRTIS-M image at $1100 \mathrm{~nm}$ (selected to show the nucleus at the same scale). The two vertical axes in each image show the operation mode of VIRTIS-M: the instrument scans spatially (number of the line on the left) and temporally (time on the right) through the coma and nucleus of the comet. The green line indicates the mid-point inside each mask for the $\pm 45^{\circ}$ sector and the full annulus.
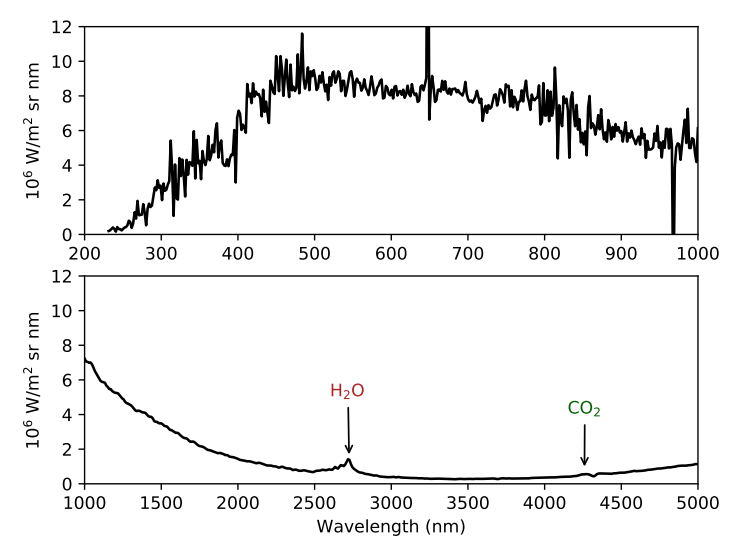

Fig. 2. Radiance spectrum for a VIRTIS-M cube in the VIS channel (V1_00388760489) (top panel) and in the IR channel (I1_00388760487) (bottom panel). The dust continuum, in the range $200-3000 \mathrm{~nm}$, is due to sunlight scattered by the dust particles in the coma. The IR spectrum shows the gas fluorescence emission of water vapor and $\mathrm{CO}_{2}$ at 2700 and $4200 \mathrm{~nm}$, respectively.

VIRTIS reduction pipeline as described by Ammannito et al. (2006), Filacchione et al. (2006), and Rinaldi et al. (2016).

A typical radiance spectrum of the 67P coma in the VIS and IR is shown in Fig. 2. It demonstrates the unique capability of the VIRTIS-M instrument to simultaneously measure the dust continuum in the range 200-3000 $\mathrm{nm}$ and the fluorescence emission of water vapor and $\mathrm{CO}_{2}$. The calculation of $\mathrm{H}_{2} \mathrm{O}$ and $\mathrm{CO}_{2}$ band intensity was described by Migliorini et al. (2016) and Fink et al. (2016).

To measure the dust continuum intensity, we chose a $9.8 \mathrm{~nm}$ wide band centered at $612.6 \mathrm{~nm}$, which corresponds to the OSIRIS WAC VIS610 filter. This allowed a comparison of the results obtained by the two instruments.

\subsection{Aperture photometry}

In each image, we measured the average signal in an annulus, or in a sector of the annulus, at fixed distance (in kilometers) from the center of the comet (Fig. 1). We call the selected area where the flux is measured "mask". We chose a mask width of $0.2 \mathrm{~km}$ in radial direction for the entire dataset. As distance we chose the maximum distance from the comet center for which the mask was fully included in the VIRTIS-M frame, which is smaller than the OSIRIS WAC FOV. For this dataset the selected distance is $3.1 \mathrm{~km}$. The irregular shape of the comet means that a single-circle mask with a fixed distance from the center of the comet is not equidistant to the limb of the comet.

Owing to the VIRTIS-M operation mode (see Sect. 2.1), each image cube line was acquired at a different time and therefore has a different longitude of the subsolar point. For each image cube, we determined the mid-point inside each mask, as shown in Fig. 1, and calculated the subsolar longitude of the mid-point. The subsolar longitude is mask dependent because the number of considered lines is different in the two masks, therefore the subsolar longitude is different for a sector or for an annulus in the same image cube (see Table 1).

For the OSIRIS images, the statistical error associated with each measurement was determined using the sigma (or error) map (Tubiana et al. 2015). It contains the error associated with the intensity of each pixel, calculated using Poisson statistics and the readout noise error. This statistical error is very small, about $\pm 0.3 \%$. In addition to this statistical error, the images have a systematic error due to the radiometric calibration of $\pm 1 \%$ (Tubiana et al. 2015). VIRTIS-M measurements have a statistical error given by the standard deviation of the average flux inside the mask and a systematic error due to the radiometric calibration. The total uncertainty on the measurement, calculated using error propagation, is about $\pm 10 \%$ (Filacchione et al. 2006; Coradini et al. 2007).

\subsection{Azimuthal profiles}

To determine the azimuthal profiles of dust and gas, we used the circular mask at $3.1 \pm 0.2 \mathrm{~km}$ from the center of the comet. The selected angular step was $10^{\circ}$. The profiles were measured clockwise. Zero degree is in the subsolar direction, as sketched in Fig. 1.

\section{Dust coma at $612 \mathrm{~nm}$}

\subsection{Longitudinal variation}

The strongest dust signal is observed in the subsolar direction (Fig. 3; see also Sect. 5). To study the overall dust coma, we selected a mask with $90^{\circ}$ angular size $\left( \pm 45^{\circ}\right)$ in the subsolar direction, as shown in Fig. 1 (right panel). We chose this angular size to minimize the contribution of fine-scale structures (i.e., jets) in the images.

Figure 4 shows the longitudinal variation of the dust flux. Each point of the figure represents the average dust flux inside the $\pm 45^{\circ}$ mask measured in OSIRIS (blue triangles) and VIRTIS-M (orange squares) images. The measured average flux in the $\pm 45^{\circ}$ mask is summarized in Table A.1.

The OSIRIS and VIRTIS-M measurements are in very good agreement at the wavelength used for the analysis (Fig. 4). In addition, the good agreement gives us the possibility to directly compare OSIRIS measurements with those of VIRTIS-M at different wavelengths, without having to consider possible instrumental effects. As described in Sect. 2.2.2, the analysis of the dust continuum in the VIRTIS-M data is limited by in-field stray light when the instrument slit is partially illuminated by the bright nucleus, as is the case in our observations. For this reason, we used only the OSIRIS images to study the dust in all subsequent sections. This avoids interpolating across stray-light regions, as was necessary in previous work (Rinaldi et al. 2016). We used VIRTIS-M to measure the gas. 


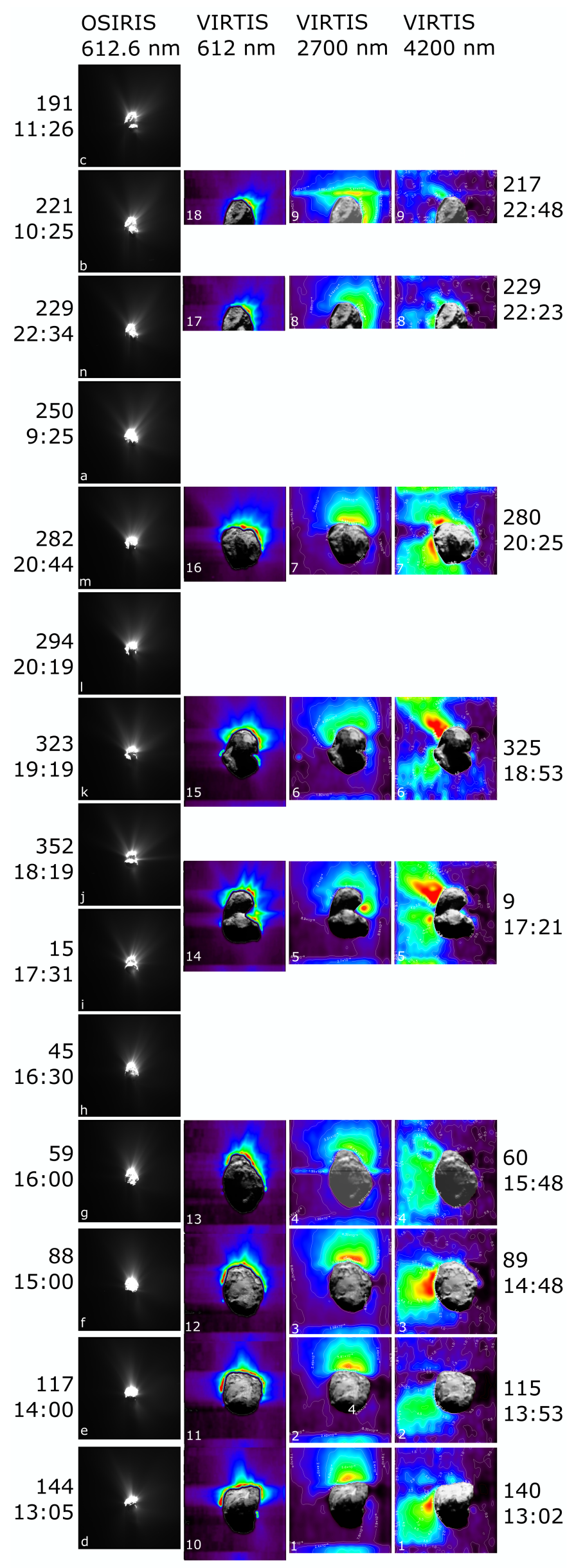

Fig. 3. OSIRIS and VIRTIS-M images. For each dataset, the images were scaled to the same brightness level and are displayed in the standard Rosetta orientation, with the Sun up. First column: OSIRIS WAC images in the VIS610 filter. On the left-hand side, the subsolar longitude (in degrees) and start time of each image (in UTC) are listed. Second column: VIRTIS-M image at $612 \mathrm{~nm}$. Third and fourth columns: band intensity maps of $\mathrm{H}_{2} \mathrm{O}$ and $\mathrm{CO}_{2}$, respectively. For better visualization, a VIRTIS-M image at $4200 \mathrm{~nm}$ was inserted into the nucleus area in the images at $612 \mathrm{~nm}, 2700 \mathrm{~nm}$, and $4200 \mathrm{~nm}$. On the right-hand side, the subsolar longitude (in degrees) and start time of each image (in UTC) are listed. Each image is labeled with the assigned number listed in Col. 1 of Tables 1 and 2. 


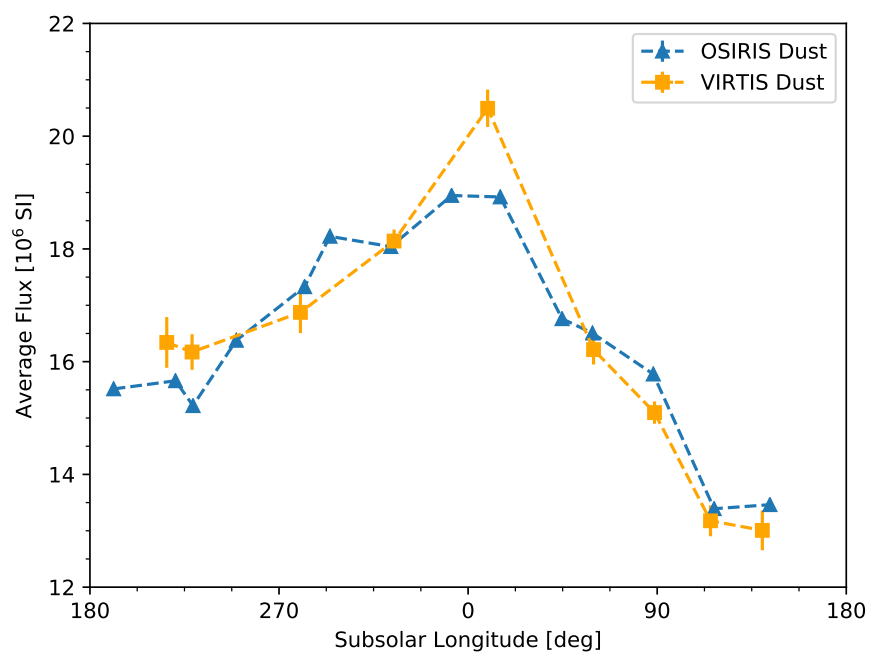

Fig. 4. Longitudinal variation of the dust flux. Each data point is the average dust flux inside the $\pm 45^{\circ}$ sector in subsolar direction at $3.1 \mathrm{~km}$ from the comet center, measured in OSIRIS (blue) and VIRTIS-M (orange) images. The error bars associated with the OSIRIS data points are too small to be discerned in the plot.
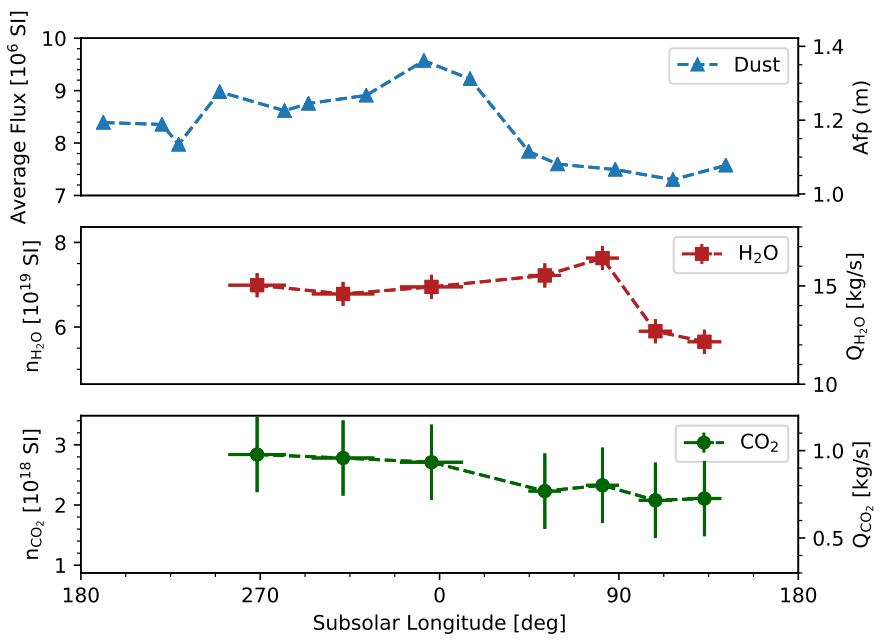

Fig. 5. Average dust flux and corresponding $A f \rho$ (top panel), $\mathrm{H}_{2} \mathrm{O}$ (central panel), and $\mathrm{CO}_{2}$ (bottom panel) column densities and corresponding production rates as functions of subsolar longitude in an annulus at $3.1 \mathrm{~km}$ from the center of the comet.

\subsection{Dust brightness and Afo}

We calculated $A f \rho$, which is commonly used to quantify dust brightness in comets (A'Hearn et al. 1984) and is often used as a proxy of dust production; it is proportional to the dust-loss rate if the dust size distribution and velocity are constant. As we do not know the size distribution or velocity, we only used the observed flux of scattered light to quantify the dust in each image, and included the conversion into $A f \rho$ only to allow convenient comparison with other observations, but did not attempt to derive any absolute dust production rate in $\mathrm{kg} \mathrm{s}^{-1}$. The average flux in a full annulus as a function of subsolar longitude is shown in Fig. 5 and summarized in Table A.1. About $50 \%$ of the total dust flux in the full annulus comes from the $90^{\circ}$ sector in subsolar direction, as shown in Table A.1.

To translate the observed scattered light intensity along the line of sight (LoS) into a local $A f \rho$, we used the method developed in Fink \& Rubin (2012) and Fink \& Rinaldi (2015). When the considered annulus is at a sufficiently large distance from the nucleus, in the collision-free flowing zone, and when no additional production or destruction of dust occurs, the calculated Af $\rho$ can provide a global measure of $A f \rho$ in the immediate vicinity of the nucleus, and it will miss only a small fraction of the total emitted dust. Rinaldi et al. (2016) found that at a distance closer than $4 \mathrm{~km}$ from the surface, the dust intensity decreases much faster than $1 / \rho$, which implies that the dust acceleration region is sampled by our measurements at $3.1 \mathrm{~km}$ from the cometary center. Gerig et al. (2018) determined that the average starting point of the $1 / \rho$ behavior is $(11.9 \pm 2.8) \mathrm{km}$. Because the steady-state condition is not fulfilled here, the determined Af $\rho$ cannot be directly compared with ground-based global measurements. Nevertheless, the result we obtain is similar to the ground-based value of $A f \rho \sim 0.9-1.0 \mathrm{~m}$ at this time (Snodgrass et al. 2016). We obtained an $A f \rho$ between $1.0 \mathrm{~m}$ and $1.4 \mathrm{~m}$, as shown in Fig. 5.

\section{Gas production rate: $\mathrm{H}_{2} \mathrm{O}$ and $\mathrm{CO}_{2}$}

\subsection{Gas production rate derived from VIRTIS-M data}

To determine the gas production rates, we calculated the average emitted band intensity inside the $3.1 \mathrm{~km}$ annulus and translated it into a gas column density $(n(\rho))$ (Migliorini et al. 2016; Fink et al. 2016). For these calculations we discarded the last image cube (\# 9) because of some radiometric problems at the wavelengths close to the $\mathrm{H}_{2} \mathrm{O}$ gas emission. In image cube \# 8 (see Fig. 3) only the dayside part of the coma is observed, so that this image cube was also discarded. Only in the first seven image cubes (\# 1-7) the full nucleus is inside the FOV, which is required for retrieving the complete azimuthal behavior of the gas in the coma.

For the combined $\mathrm{H}_{2} \mathrm{O}$ bands at $2660 \mathrm{~nm}$ and $2730 \mathrm{~nm}$ we used the fluorescence efficiency at 1 au $g_{0}=2.745 \times$ $10^{23} \mathrm{~W} \mathrm{molec}{ }^{-1}$ (Bockelée-Morvan et al. 2015), and for the $\mathrm{CO}_{2}$ band we used $g_{0}=1.25 \times 10^{22} \mathrm{~W} \mathrm{molec}^{-1}$ (Debout et al. 2016). The uncertainty on the $\mathrm{H}_{2} \mathrm{O}$ and $\mathrm{CO}_{2}$ column density calculations is $\sim 10 \%$. The measured average water and $\mathrm{CO}_{2}$ column densities as functions of subsolar longitude are displayed in Fig. 5 (center and bottom panels) and summarized in Table A.1. At $3.1 \mathrm{~km}$ from the comet center, $78 \%$ of the water column density is contained within an angle of $\pm 90^{\circ}$ and $52 \%$ within an angle of $\pm 45^{\circ}$ in the subsolar direction. There is very little scatter in the percentages in the first seven observations despite the different configurations of nucleus, Sun, and spacecraft. The $\mathrm{CO}_{2}$ distribution does not follow the direct solar illumination and has essentially no correlation with the water column density distribution (Fig. 5). The $\mathrm{CO}_{2}$ molecules are emitted mostly from the southern hemisphere of the comet, as shown in Fig. 3 (fourth column). This is the reason why $50 \%$ of the $\mathrm{CO}_{2}$ column density is contained within an angle of $\pm 90^{\circ}$ in the subsolar direction (day side) and the same percentage is found on the night side, with little variability in these percentages (Table A.1). The large variability of the $\mathrm{CO}_{2}$ column density, from 19 to $38 \%$, in the $\pm 45^{\circ}$ sector in subsolar direction is due to the orientation of the cometary spin axis during these observations.

To convert the gas column densities into production rates, we used the method described in Fink et al. (2016). The gas production rates are listed in Table A.2. For the same dataset, Fink et al. (2016) analyzed the emission intensity of $\mathrm{CO}_{2}$ and $\mathrm{H}_{2} \mathrm{O}$ and their distribution in the coma using a slightly different annulus at $2.8 \mathrm{~km}$ from the center to the comet. Our result obtained here for the $\mathrm{H}_{2} \mathrm{O}$ and $\mathrm{CO}_{2}$ distribution agrees well with the findings of 


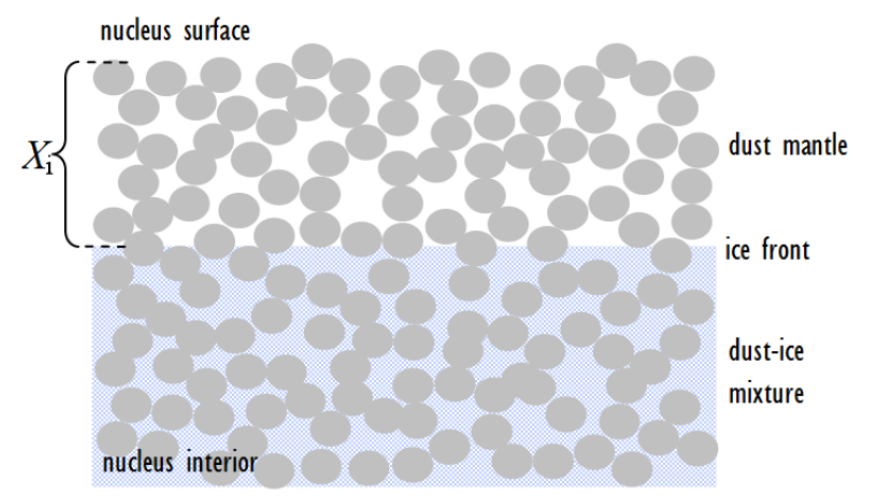

Fig. 6. Illustration of the dust cover assumed in the thermophysical model. Figure adapted from Hu et al. (2017b). $X_{i}$ is the thickness of the desiccated dust mantel.

Fink et al. (2016) and the gas spatial distribution maps obtained by Migliorini et al. (2016).

Figure 5 shows that there is little correlation between the measured productions of dust, water, and $\mathrm{CO}_{2}$. In addition, none of the patterns corresponds to the variation of the cross-section area of the illuminated nucleus (Fig. 7 bottom panel). The lack of correlation between dust and $\mathrm{CO}_{2}$ is not surprising, indicating that $\mathrm{CO}_{2}$ outgassing does not play a dominant role in driving the global dust emission. On the other hand, the discrepancy between the patterns of dust and water measurements is harder to explain. Water outgassing is the dominant driver of dust activity from the northern hemisphere (De Sanctis et al. 2015; Shi et al. 2018a) before the observations we analyzed here (days before northern autumn equinox). The deviation of water production from the variation of the illuminated cross section, in particular, suggests that the nucleus surface is not homogeneously active, as presented by Marschall et al. (2019). In the following section, we perform a simple thermophysical analysis in order to shed some light on this discrepancy.

\section{2. $\mathrm{H}_{2} \mathrm{O}$ production rate computed with a thermophysical model}

We wish to compare the observed pattern of dust and gas release to a simplified model that describes what would be expected in the case of homogenous activity that is only controlled by illumination of each surface element. In order to do this, we employed a thermophysical model ( $\mathrm{Hu}$ et al. 2017a) to estimate the total water production rate of the nucleus over a full rotation of $67 \mathrm{P}$ that encompasses all observations described above.

\subsubsection{Model description}

The shape of the nucleus is approximated by a model consisting of 1500 facets (Preusker et al. 2015). We assumed that the nucleus is covered by a desiccated dust layer, or dust mantle, of constant thickness that is composed of uniform spherical dust aggregates. Water ice is present underneath the dust mantle (Fig. 6). The sublimation flux is strongly influenced by the temperature of the ice front. The temperatures of the nucleus subsurface as a function of depth are estimated by solving the 1D heat equation, balancing the input energy from solar illumination of the surface with heat that is reradiated, conducted into the surface, or used in sublimating ice.

However, the interior of the nucleus (beneath the mantle) is not composed of pure water ice, and sublimation can therefore
Table 3. Parameters for thermophysical modeling.

\begin{tabular}{lcr}
\hline \hline Parameter & Symbol & Value \\
\hline Bond albedo & $A_{\mathrm{B}}$ & 0.01 \\
Emissivity & $\varepsilon$ & 1 \\
Heat conductivity $\left(\mathrm{W} \mathrm{m}^{-1} \mathrm{~K}^{-1}\right)$ & $\kappa$ & $2 \times 10^{-3}$ \\
Specific heat capacity $\left(\mathrm{J} \mathrm{kg}^{-1} \mathrm{~K}^{-1}\right)$ & $c$ & 1000 \\
Density $\left(\mathrm{kg} \mathrm{m}^{-3}\right)$ & $\varrho$ & 500 \\
Diameter of dust aggregate $(\mathrm{mm})$ & $d_{\mathrm{P}}$ & 1 \\
Thickness of dust mantle $(\mathrm{mm})$ & $X_{\mathrm{i}}$ & 5 \\
Area fraction of ice & $f_{\mathrm{i}}$ & 0.01 \\
\hline
\end{tabular}

not take place everywhere. For this reason, the factor $f_{\mathrm{i}} \in(0,1)$ is introduced. It formally measures the areal fraction of water ice and is approximately inverse to the dust-to-ice ratio of the subsurface (Crifo 1997). We assumed that heat flux vanishes beyond several (diurnal) skin depths.

At any given epoch, the position vector of the Sun with respect to (the body-fixed frame of) the nucleus is obtained through the SPICE kernels ${ }^{2}$ for $67 \mathrm{P}$. It is subsequently transformed into local horizontal coordinates through a series of rotations of the coordinate system to yield the solar incidence angle. We made use of a "Landscape" database for 67P that delineates the skyline at each location on the nucleus (each facet of the shape model) in order to efficiently determine the local illumination (Hu et al. 2017a).

The 1D heat equation is solved with the Crank-Nicolson method. The solutions are diurnally equilibrated temperatures $T$ and water production rates $Z$ that repeat or coincide, if the seasonal cycle is neglected, with the rates exactly one comet rotation apart, for example, $T\left(t \pm t_{\mathrm{p}}\right)=T(t)$ and $Z\left(t \pm t_{\mathrm{p}}\right)=Z(t)$, where $t_{\mathrm{P}}$ is the rotation period of $67 \mathrm{P}$.

\subsubsection{Choice of model parameters}

A summary of the key parameters of the thermophysical model is given in Table 3. For simplicity, it is assumed that the nucleus subsurface is homogeneous. We also neglect variability of the parameters, for instance, changes in mantle thickness and loss of ice underneath. With these assumptions, the model parameters are treated as constants. Following the argument by Blum et al. (2017) that the dust aggregates, clearly nonuniform in size, are about a few millimeter in diameter, we adopted a diameter of the dust aggregates of $d_{\mathrm{P}}=1 \mathrm{~mm}$. The OSIRIS observation of the dust activity that continued for about one hour after sunset indicates that water ice was present at some depth of less than $1 \mathrm{~cm}$ (Shi et al. 2016). The long-term (seasonal) evolution of the total water production of $67 \mathrm{P}$ throughout perihelion can also be modeled with a mantle thickness of $5 \leq X_{\mathrm{i}} \leq 10 \mathrm{~mm}$. Overall, water ice is rarely exposed (Capaccioni et al. 2015). When detected, it is usually present in small quantities, for example, a few percent (De Sanctis et al. 2015; Filacchione et al. 2016a; Barucci et al. 2016). There is observational evidence that the average water-ice abundance in the top about $1 \mathrm{~m}$ above the northern hemisphere cannot exceed 10\% (Hu et al. 2017b). Therefore, the area fraction of water ice $\left(f_{\mathrm{i}}\right)$ is probably similar. The scarcity of ice suggests that the thermophysical properties of the nucleus are dominated by those of the refractory component. We are aware that the thermal parameters, that is, the conductivity $(\kappa)$

2 The SPICE kernels are available from ESA at https://www. cosmos.esa.int/web/spice/spice-for-rosetta 


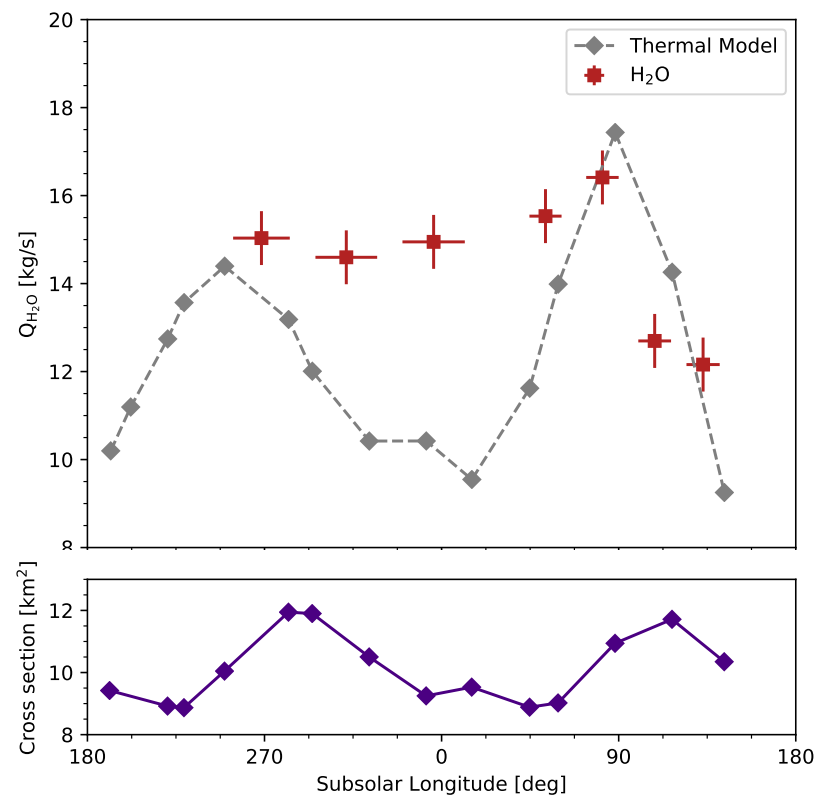

Fig. 7. Top panel: comparison between the water production rate as output of the thermophysical model and the rate measured in VIRTIS-M-IR spectra. Bottom panel: illuminated nucleus cross-section at the time of each OSIRIS observation.

and specific heat capacity (c), are dependent on temperature. In particular, thermal radiation through the pores enhances the efficiency of heat transfer. In this case, the radiative component of the conductivity varies with $T^{3}$. However, the radiation most significantly affects temperatures below the diurnal skin. In the case of millimeter-sized particles and when ice sublimation is assumed to occur mostly from above the diurnal skin depth, the enhancement of water production by radiation is not notable ( $\mathrm{Hu}$ et al. 2019). In addition, the dependence of heat capacity on temperature is linear (Orosei et al. 1995), while the exact behavior of material on 67P is largely unknown. We therefore neglected the temperature dependence of the parameters and adopted $\kappa_{\mathrm{d}}=\kappa_{\mathrm{i}}=0.002 \mathrm{~W} \mathrm{~m}^{-1} \mathrm{~K}^{-1}$ (conductivity of the dry dust mantle and the underlying icy dust, respectively), $c=1000 \mathrm{~J} \mathrm{~kg}^{-1} \mathrm{~K}^{-1}$ and $\varrho=500 \mathrm{~kg} \mathrm{~m}^{-3}$, which corresponds to a thermal inertia of $30 \mathrm{~W} \mathrm{~m}^{-2} \mathrm{~K}^{-1} \mathrm{~s}^{1 / 2}$, as measured by MIRO (Schloerb et al. 2015).

\subsubsection{Model results}

The water production rates at each observation time from the output of the thermal model and the VIRTIS-M observations described in Sect. 4.1 are shown in Fig. 7. Regulated by the mantle thickness and ice abundance, the modeled water production rate agrees well with the measurements in terms of overall magnitude. The variation largely follows that of the cross-section area of the illuminated nucleus, as expected for a model of homogeneous nucleus activity. A phase shift of about $20^{\circ}$ in subsolar longitude is evidently attributable to the presence of the dust mantle, which causes a thermal lag of about half an hour at the depth of the ice front (i.e., $5 \mathrm{~mm}$ ). However, the modeled production rate is clearly underestimated compared with the measurements in the subsolar longitude range $\left(270^{\circ}-50^{\circ}\right)$. This indicates that the real gas production varies between areas and must depend on local (sub-)surface properties, not just illumination and topography of the nucleus; this is discussed further below.
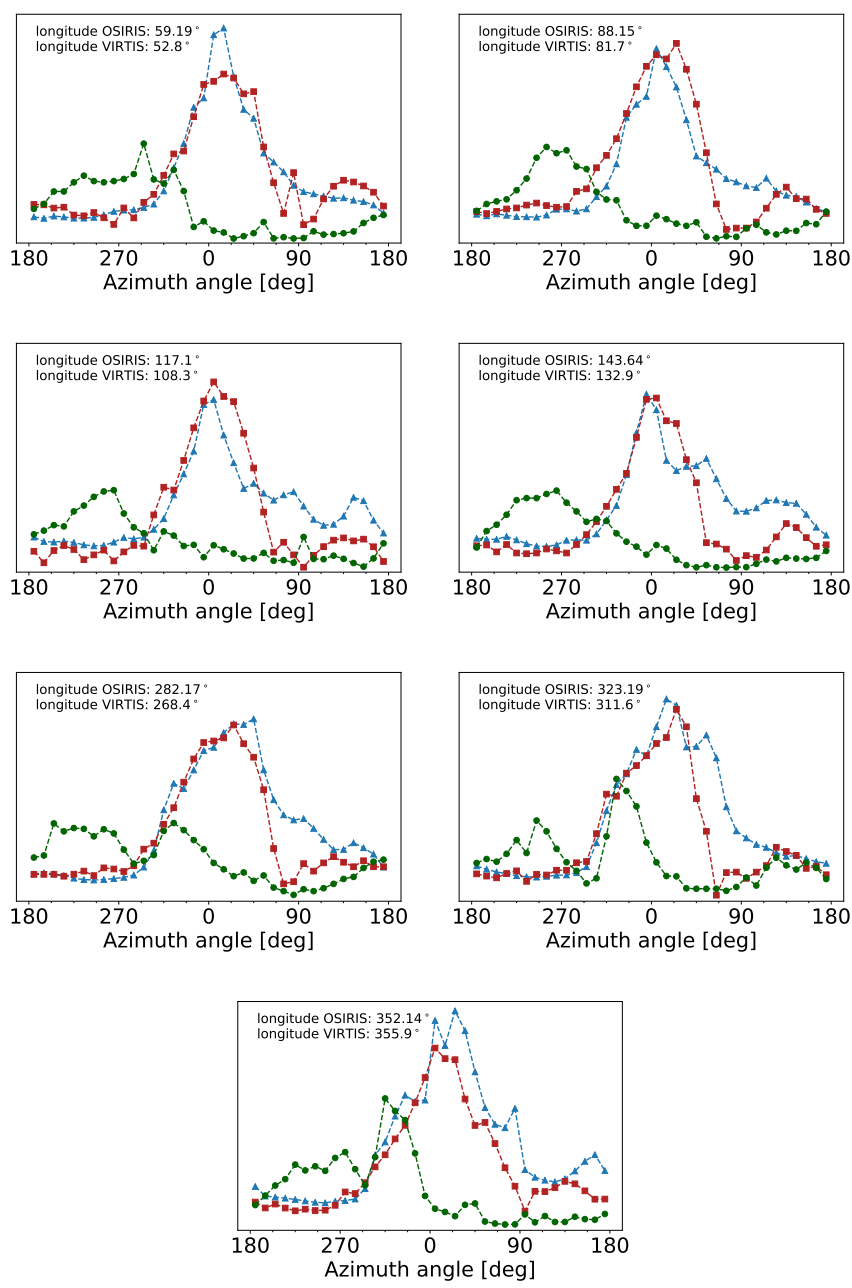

Fig. 8. Azimuthal profiles of dust brightness and gas band intensity. OSIRIS dust is shown as blue triangles, VIRTIS- $\mathrm{M} \mathrm{H}_{2} \mathrm{O}$ as red squares, and VIRTIS-M $\mathrm{CO}_{2}$ as green circles. For displaying purposes, the VIRTIS- $\mathrm{M} \mathrm{H}_{2} \mathrm{O}$ and $\mathrm{CO}_{2}$ band area intensities are scaled to the OSIRIS dust values.

\section{Azimuthal gas and dust distribution}

Figure 8 shows the angular distribution of water (red squares) and $\mathrm{CO}_{2}$ (green circles) band intensity, measured in the first seven VIRTIS-M image cubes, and the angular distribution of the dust brightness (blue triangles), measured in the OSIRIS images that are closest in the subsolar longitude to the VIRTIS-M data. In all observations, the absolute maximum for dust and water vapor is located in the subsolar direction. A secondary water vapor peak occurs at roughly $130^{\circ}$ and is related to the variable illumination of the neck area. $\mathrm{CO}_{2}$ peaks between $180^{\circ}$ and $270^{\circ}$, consistent with the southern hemisphere illuminated (Fig. 3).

The spatial (or angular) correlation between dust and water, both coming from the subsolar side of the comet, which has previously been observed by Rinaldi et al. (2016), shows that water is the main driver of dust activity in this time period. This is also generally consistent with observations from the ground, showing that long-term variations in total water production rate correlate with the total dust brightness (Hansen et al. 2016).

The presence of $\mathrm{CO}_{2}$ ice in these regions of the southern hemisphere and in the same period is not surprising because VIRTIS detected a $\mathrm{CO}_{2}$-ice rich area in the Anhur region at the 


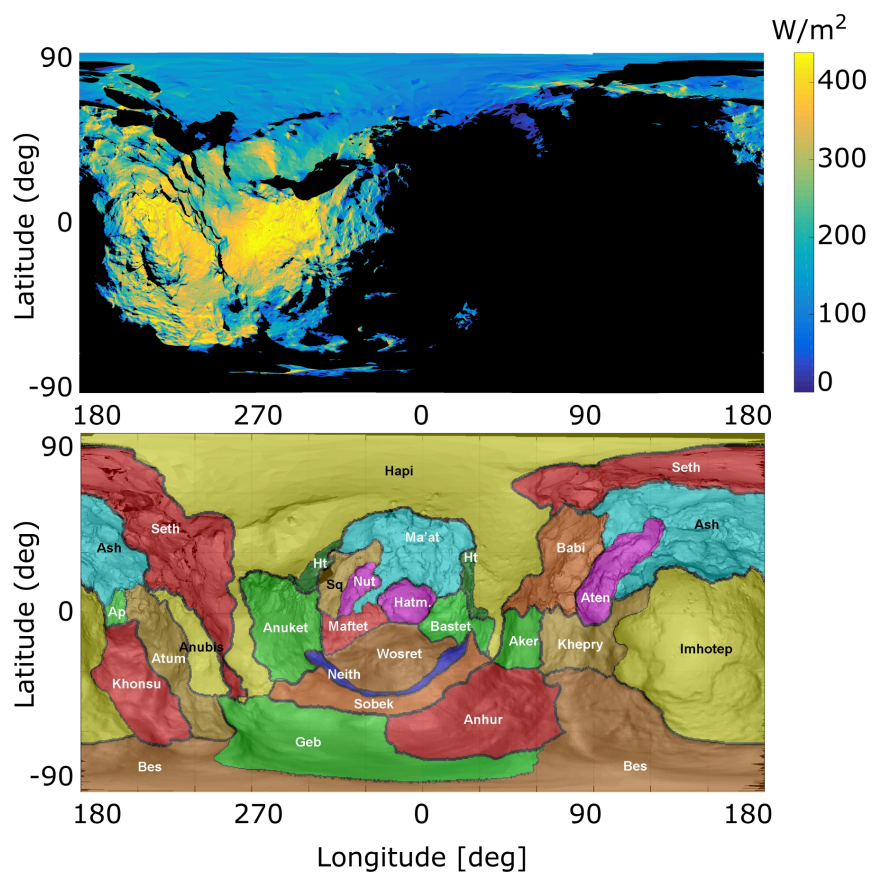

Fig. 9. Top panel: example of an insolation map, calculated for 09:25:32 UTC. Bottom panel: region map of 67P adapted from El-Maarry et al. (2016).

end of March 2015 (Filacchione et al. 2016b). In the southern hemisphere, the $\mathrm{CO}_{2}$ ice is generally closer to the surface and therefore more easily accessible. When the comet approaches the Sun, the southern summer is very intense. In this strong sunlight, erosion rates are fast enough to expose fresh primordial layers of the interior, which are rich in $\mathrm{CO}_{2}$. Quantitative calculations of the comet erosion rates during its orbit are presented by Keller et al. (2015). The increase in $\mathrm{CO}_{2}$ abundance in the coma during the perihelion time-frame is analyzed in Bockelée-Morvan et al. (2016). The authors found that during this period, the measured abundance ratios of $\mathrm{CO}_{2}$ increased by a factor of 30 with respect to what was found above the illuminated northern hemisphere.

\section{Discussion}

To understand the illumination conditions across the surface of the nucleus of $67 \mathrm{P}$ at the time of each OSIRIS observation, we generated insolation maps. One example is shown in Fig. 9 (top panel), along with a map in which the different regions of the nucleus are identified (bottom panel). The distribution of solar irradiance is calculated on a polyhedral shape model representing the nucleus of 67P with 499902 facets (Preusker et al. 2017). For each epoch, the Sun's position in the Cheops bodyfixed frame of the comet (as defined in Preusker et al. 2015) was derived from the reconstructed ephemeris and rotational status of 67P using the SPICE tool-kit (Acton 1996). The concave shape of $67 \mathrm{P}$ prevents the display of certain surface areas in maps with equidistant cylindrical projection. However, the overall illumination pattern shown in the figure is not affected.

Figure 10 displays the insolation maps at the time of each OSIRIS observation. In the central inset we show the modeled and observed $\mathrm{H}_{2} \mathrm{O}$ production rates (gray and red curves, respectively), the observed $\mathrm{CO}_{2}$ production rate (green curve), and the total dust brightness (blue curve), calculated inside the full $360^{\circ}$ annulus (see Table A.2). There is no strong temporal correlation between total dust brightness and water production rates (neither observed nor from the simple model). It should be emphasized (as noted in Sect. 5), however, that water is still the main driver of dust activity in this time period. Only the ratio between water production and dust activity changes.

The green box in Fig. 10 highlights the excess of water production (red curve) compared to the simple homogeneous model (gray curve) at subsolar longitudes between $270^{\circ}$ and $50^{\circ}$. The green lines emphasize the corresponding insolation maps. This corresponds to epochs when the head lobe and regions of the southern hemisphere with strong seasonal variations (e.g., Bes, Geb, Anhur) are illuminated. While we caution against over interpreting the differences between our simple thermal model and the observed water production, it could be argued that these are most easily explained by a higher activity of these southern regions with respect to the northern regions.

The observed dust brightness (blue curve) shows a pronounced maximum around $0^{\circ}$ subsolar longitude, which is not pronounced in the water production (red curve). Previous studies have already shown that the Anhur and Bes regions, which are illuminated at this time, are highly active and sources of several jets (Vincent et al. 2016; Fornasier et al. 2017), thus in agreement with our findings.

At the same epoch (green region) there is also a maximum of $\mathrm{CO}_{2}$. The analysis of the azimuthal profiles in Sect. 5 (Fig. 8) showed that the dust is correlated with water and not $\mathrm{CO}_{2}$. The increased $\mathrm{CO}_{2}$ production in this epoch is therefore not responsible for the peak in the dust activity.

The largest discrepancy between dust and gas production rates can be observed in the red box of Fig. 10. The dust brightness drastically decreases in this subsolar longitude range $\left(50^{\circ}-90^{\circ}\right)$, while instead the water production (measured and from the model) displays a maximum. These epochs correspond to when northern consolidated regions (e.g., Bastet, Aker, Khepry, Aten, Babi; Thomas et al. 2018; see Fig. 9, bottom panel) are illuminated and the southern hemisphere regions with strong seasons are in shadow.

This temporal noncorrelation (red box) can either be explained by regional variations of surface properties or regional variations in the scattering properties of the lifted dust particles. More specifically, we discuss here the effect of regional variations in (a) thickness of the desiccated layer, (b) surface cohesion, and the presence of large particles that affect (c) gas coupling, thus lifting, and (d) permeability of the dust layer. For the scattering properties of the dust particles, we discuss (e) the composition of lifted dust particles and (f) their sizes.

(a) We do not attribute the discrepancy of dust and gas production in the red box to the thickness of the desiccated layer on the nucleus surface because a thicker layer would also quench gas production. (b) A higher cohesion of surface material could quench dust activity (Bischoff et al. 2019), where the water vapor would escape without lifting dust. This is possible and likely in consolidated regions, which consist of centimeter-sized pebbles. (c) Even if large (decimeter- to meter-sized) particles were easily lifted against their cohesion, they still carry a high mass inertia. Their low size-to-mass ratio could prevent large enough particles from being carried into the coma from the gas drag. Fallback for particles in the considered size range was observed by Agarwal et al. (2016), and many more of these might not even be considerably lifted. (d) If the upper dust layer is dominated by large particles, the gas permeability would also be enhanced. For granular materials with macroscopic voids, Gundlach et al. (2011) have shown that the gas permeability increases with the size of the constituent particles. A layer of decimeter-sized dust 


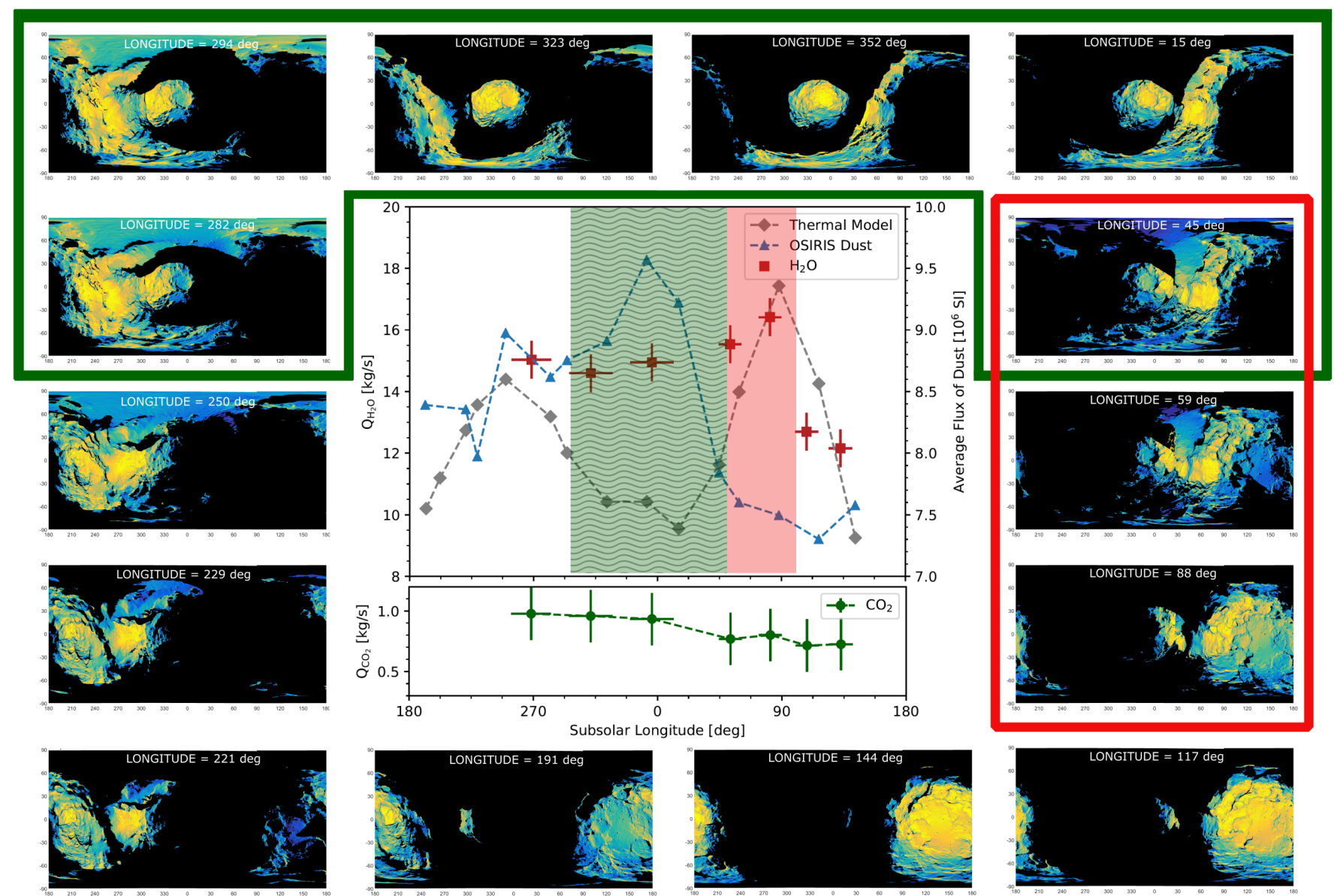

Fig. 10. Insolation maps for the time of each OSIRIS observation. Inset: total dust brightness and gas production rates, measured in the full $3.1 \mathrm{~km}$ annulus, as functions of subsolar longitude. The green box encloses the insolation maps that correspond to the subsolar longitudes where an excess of measured water production was found, relative to a simple model. The red box frames the insolation maps that correspond to the subsolar longitudes where a minimum of dust and a maximum of water production were measured.

particles (cf. Pajola et al. 2017) would thereafter have a ten times higher permeability than a layer of the same thickness of centimeter-sized particles. A higher gas permeability of the dust layer would result in a reduced pressure buildup and thus reduced dust production. The gas could simply escape through the large voids. Decimeter- to meter-sized particles, which are present in fallback regions (e.g., Ma'at), are almost entirely cleaned up in consolidated regions, which exposes the underlying consolidated material. This is consistent with the idea of "self-cleaning" of the northern hemisphere proposed by Fulle et al. (2019). (c) and (d) could quench dust activity in fallback regions, but do not play a role in consolidated areas.

For the coma-related effects (e and $\mathrm{f}$ ), it is worth repeating that the observed dust brightness (or equivalently $A f \rho$ ) is proportional to the dust-loss rate only if the dust velocity, size distribution, and composition do not change. A variation in dust brightness might therefore in principle be interpreted as a change in dust-loss rate or as a change in any of the parameters above. (e) We rule out significant differences in the dust properties in different areas (i.e., at different times) due to composition variation, as none was observed in VIRTIS coma observations (Rinaldi et al. 2016). (f) If the coma were dominated by larger particles, they would tend to reduce the observed dust brightness for the same dust production rate in $\mathrm{kg} \mathrm{s}^{-1}$ because the fewer but larger particles would constitute a smaller reflecting area. This would play a role in fallback regions, but not in the consolidated regions that we considered. Measurements of the dust size distribution from the in situ instruments on board Rosetta are difficult to separate by different surface areas as they were measured over extended periods, during which the sub-spacecraft location changed considerably, but there are hints of variation. For example, GIADA detected more compact particles from Hapi (a fallback region) and more fluffy aggregates elsewhere (Della Corte et al. 2015), suggesting variations in the size distribution; but again, this does not demonstrate differences between different consolidated terrains. Differences were observed in size distribution in the dust that was released during outbursts relative to the background coma, but this may be related to the (poorly understood) outburst process rather than regional differences (Bockelée-Morvan et al. 2017). In summary, the best explanation of our observations in the red box in Fig. 10 is a quenched dust activity due to high cohesion of surface material typical of consolidated regions.

The observations in the green box in Fig. 10, an increased water activity in the southern regions with respect to model expectations, are likely to be attributable to regional changes in volatile content or access to these. Our simple thermal model assumes the same thickness of the desiccated layer for the northern and southern hemisphere. The erosion rates in the south are higher, therefore this is likely not the case, and a shallower desiccated layer or a larger area fraction of ice (Fougere et al. 2016) would explain our observations. 
A more thorough investigation in the near future requires applying more sophisticated thermophysical models to treat not only nonuniform properties of the nucleus subsurface, but also activity of multiple volatile species. It would be necessary to resolve the temperatures in layers deeper than the diurnal skin depth as considered in the current study. A more detailed characterization of the physical processes in the subsurface, such as phase change and mass transfer of volatiles as well as the resulting material loss, is also desired, as the phenomena already proved to influence the energy budget of the system strongly (de Sanctis et al. 1999; Capria et al. 2000; Prialnik et al. 2004; Gortsas et al. 2011).

\section{Summary and conclusion}

We have analyzed one OSIRIS and one VIRTIS-M dataset acquired on 27 April 2015, when the comet was at 1.76 au from the Sun in the inbound arc. No strong temporal correlation between total dust brightness and water production rates is found, although water is still the main driver of dust activity at this period in time. The observed increased water activity in the southern regions with strong seasonal variations with respect to model expectations is likely to be attributable to regional changes in volatile content or access to this. The best explanation for the drastic decrease in dust brightness when consolidated regions are illuminated is a quenched dust activity due to the high cohesion of surface material. These observations show that when $67 \mathrm{P}$ is approaching perihelion, the dust activity cannot be understood based on water-driven activity alone. This is in agreement with other modeling results on the seasonal evolution of the near-nucleus coma, which show that the correlation that was observed earlier in the mission between the observed dust coma and a modeled water coma from a homogeneously sublimating nucleus is significantly degraded (Shi et al. 2018b).

Acknowledgements. OSIRIS was built by a consortium led by the MaxPlanck-Institut für Sonnensystemforschung, Göttingen, Germany, in collaboration with CISAS, University of Padova, Italy, the Laboratoire d'Astrophysique de Marseille, France, the Instituto de Astrofísica de Andalucia, CSIC, Granada Spain, the Scientific Support Office of the European Space Agency, Noordwijk, The Netherlands, the Instituto Nacional de Técnica Aeroespacial, Madrid, Spain, the Universidad Politéchnica de Madrid, Spain, the Department of Physics and Astronomy of Uppsala University, Sweden, and the Institut für Datentechnik und Kommunikationsnetze der Technischen Universität Braunschweig, Germany. The support of the national funding agencies of Germany (DLR), France (CNES), Italy (ASI), Spain (MEC), Sweden (SNSB), and the ESA Technical Directorate is gratefully acknowledged. We thank the Rosetta Science Ground Segment at ESAC, the Rosetta Mission Operations Centre at ESOC and the Rosetta Project at ESTEC for their outstanding work enabling the science return of the Rosetta Mission. VIRTIS was built by a consortium, which includes Italy, France, and Germany, under the scientific responsibility of the Istituto di Astrofisica e Planetologia Spaziali of INAF, Italy, which also guides the scientific operations. The VIRTIS instrument development, led by the prime contractor Leonardo-Finmeccanica (Florence, Italy), has been funded and managed by ASI, with contributions from Observatoire de Meudon financed by CNES, and from DLR. We thank the Rosetta Science Ground Segment and the Rosetta Mission Operations Centre for their support throughout all the phases of the mission. The VIRTIS calibrated data will be available through the ESA's Planetary Science Archive Website (www.rssd.esa.int) and is available upon request until posted to the archive. We thank the following institutions and agencies for support of this work: Italian Space Agency (ASI, Italy) contract number I/024/12/1, Centre National d'Études Spatiales (CNES, France), DLR (Germany), NASA (USA) Rosetta Program, and Science and Technology Facilities Council (UK).

\section{References}

Acton, C. H. 1996, Planet. Space Sci., 44, 65

Agarwal, J., A'Hearn, M. F., Vincent, J.-B., et al. 2016, MNRAS, 462, S78

A'Hearn, M. F., Schleicher, D. G., Millis, R. L., Feldman, P. D., \& Thompson, D. T. 1984 , AJ, 89, 579
Ammannito, E., Filacchione, G., Coradini, A., et al. 2006, Rev. Sci. Instrum., 77, 093109

Attree, N., Jorda, L., Groussin, O., et al. 2019, A\&A, 630, A18 (Rosetta 2 SI) Barucci, M. A., Filacchione, G., Fornasier, S., et al. 2016, A\&A, 595, A102

Bischoff, D., Gundlach, B., Neuhaus, M., \& Blum, J. 2019, MNRAS, 483, 1202

Blum, J., Gundlach, B., Krause, M., et al. 2017, MNRAS, 469, S755

Bockelée-Morvan, D., Debout, V., Erard, S., et al. 2015, A\&A, 583, A6

Bockelée-Morvan, D., Crovisier, J., Erard, S., et al. 2016, MNRAS, 462, S170

Bockelée-Morvan, D., Rinaldi, G., Erard, S., et al. 2017, MNRAS, 469, S443

Capaccioni, F., Coradini, A., Filacchione, G., et al. 2015, Science, 347, aaa0628 Capria, M. T., Coradini, A., De Sanctis, M. C., \& Orosei, R. 2000, AJ, 119, 3112

Coradini, A., Capaccioni, F., Drossart, P., et al. 2007, Space Sci. Rev., 128, 529 Crifo, J. F. 1997, Icarus, 130, 549

de Sanctis, M. C., Capaccioni, F., Capria, M. T., et al. 1999, Planet. Space Sci., 47,855

De Sanctis, M. C., Capaccioni, F., Ciarniello, M., et al. 2015, Nature, 525, 500

Debout, V., Bockelée-Morvan, D., \& Zakharov, V. 2016, Icarus, 265, 110

Della Corte, V., Rotundi, A., Fulle, M., et al. 2015, A\&A, 583, A13

El-Maarry, M. R., Thomas, N., Gracia-Berná, A., et al. 2016, A\&A, 593, A110

Filacchione, G., Ammannito, E., Coradini, A., et al. 2006, Rev. Sci. Instrum., 77, 103106

Filacchione, G., de Sanctis, M. C., Capaccioni, F., et al. 2016a, Nature, 529, 368

Filacchione, G., Raponi, A., Capaccioni, F., et al. 2016b, Science, 354, 1563

Fink, U., \& Rubin, M. 2012, Icarus, 221, 721

Fink, U., \& Rinaldi, G. 2015, Icarus, 257, 9

Fink, U., Doose, L., Rinaldi, G., et al. 2016, Icarus, 277, 78

Fornasier, S., Feller, C., Lee, J.-C., et al. 2017, MNRAS, 469, S93

Fougere, N., Altwegg, K., Berthelier, J.-J., et al. 2016, A\&A, 588, A134

Fulle, M., Blum, J., Green, S. F., et al. 2019, MNRAS, 482, 3326

Gasc, S., Altwegg, K., Balsiger, H., et al. 2017, MNRAS, 469, S108

Gerig, S.-B., Marschall, R., Thomas, N., et al. 2018, Icarus, 311, 1

Glassmeier, K.-H., Boehnhardt, H., Koschny, D., Kührt, E., \& Richter, I. 2007, Space Sci. Rev., 128, 1

Gortsas, N., Kührt, E., Motschmann, U., \& Keller, H. U. 2011, Icarus, 212, 858 Gundlach, B., Skorov, Y. V., \& Blum, J. 2011, Icarus, 213, 710

Hansen, K. C., Altwegg, K., Berthelier, J.-J., et al. 2016, MNRAS, 462, S491

Hu, X., Shi, X., Sierks, H., et al. 2017a, MNRAS, 469, S295

Hu, X., Shi, X., Sierks, H., et al. 2017b, A\&A, 604, A114

Hu, X., Gundlach, B., von Borstel, I., Blum, J., \& Shi, X. 2019, A\&A, 630, A5 (Rosetta 2 SI)

Keller, H. U., Barbieri, C., Lamy, P., et al. 2007, Space Sci. Rev., 128, 433

Keller, H. U., Mottola, S., Davidsson, B., et al. 2015, A\&A, 583, A34

Kramer, T., \& Noack, M. 2016, ApJ, 823, L11

Kramer, T., Läuter, M., Rubin, M., \& Altwegg, K. 2017, MNRAS, 469, S20

Marschall, R., Rezac, L., Kappel, D., et al. 2019, Icarus, 328, 104

Migliorini, A., Piccioni, G., Capaccioni, F., et al. 2016, A\&A, 589, A45

Orosei, R., Capaccioni, F., Capria, M. T., et al. 1995, A\&A, 301, 613

Pajola, M., Lucchetti, A., Fulle, M., et al. 2017, MNRAS, 469, S636

Preusker, F., Scholten, F., Matz, K.-D., et al. 2015, A\&A, 583, A33

Preusker, F., Scholten, F., Matz, K.-D., et al. 2017, A\&A, 607, L1

Prialnik, D., Benkhoff, J., \& Podolak, M. 2004, Modeling the Structure and

Activity of Comet Nuclei, eds. M. C. Festou, H. U. Keller, \& H. A. Weaver

(Tucson: University of Arizona Press), 359

Rinaldi, G., Fink, U., Doose, L., et al. 2016, MNRAS, 462, S547

Schloerb, F. P., Keihm, S., von Allmen, P., et al. 2015, A\&A, 583, A29

Shi, X., Hu, X., Sierks, H., et al. 2016, A\&A, 586, A7

Shi, X., Hu, X., Mottola, S., et al. 2018a, Nat. Astron., 2, 562

Shi, X., Rose, M., \& the OSIRIS team. 2018b, European Planetary Science Congress, 12, EPSC2018

Snodgrass, C., Opitom, C., de Val-Borro, M., et al. 2016, MNRAS, 462, S138

Thomas, N., El Maarry, M. R., Theologou, P., et al. 2018, Planet. Space Sci., 164, 19

Tubiana, C., Güttler, C., Kovacs, G., et al. 2015, A\&A, 583, A46

Vincent, J.-B., A'Hearn, M. F., Lin, Z.-Y., et al. 2016, MNRAS, 462, S184

Whipple, F. L. 1950, ApJ, 111, 375

Zakharov, V. V., Ivanovski, S. L., Crifo, J.-F., et al. 2018, Icarus, 312, 121

1 Max Planck Institute for Solar System Research, Göttingen, Germany

e-mail: tubiana@mps .mpg.de

2 Istituto di Astrofisica e Planetologia Spaziali, Istituto Nazionale di Astrofisica, Rome, Italy 
${ }^{3}$ Institute for Astronomy, University of Edinburgh, Royal Observatory, Edinburgh EH9 3HJ, UK

${ }^{4}$ Institute for Geodesy and Geoinformation Science, Technical University Berlin, Straße des 17. Juni 135, 10623 Berlin, Germany

5 International Space Science Institute, Bern, Switzerland

${ }^{6}$ INAF - Osservatorio Astronomico, Trieste, Italy

${ }^{7}$ LESIA, Observatoire de Paris, PSL Research University, CNRS, Sorbonne, Université, Université Paris Diderot, Sorbonne Paris Cité, 5 place Jules Janssen, 92195 Meudon, France

${ }^{8}$ Department of Physics, Oxford University, Oxford, UK

9 Deutsches Zentrum für Luft- und Raumfahrt (DLR), Institut für Planetenforschung, Berlin, Germany

${ }^{10}$ Lunar and Planetary Laboratory, University of Arizona, Tucson, USA

${ }^{11}$ Physics Department, Auburn University, Auburn, AL 36849, USA

12 Laboratoire Atmosphères, Milieux et Observations Spatiales, CNRS \& Université de Versailles Saint-Quentin-en-Yvelines, 11 boulevard d'Alembert, 78280 Guyancourt, France

13 Centro de Astrobiologia, CSIC-INTA, Torrejon de Ardoz, Madrid, Spain

14 Science Support Office, European Space Research and Technology Centre/ESA, Keplerlaan 1, Postbus 299, 2201 AZ Noordwijk ZH, The Netherlands

15 Jet Propulsion Laboratory, M/S 183-401, 4800 Oak Grove Drive, Pasadena, CA 91109, USA

${ }^{16}$ LATMOS, CNRS/UVSQ/IPSL, 11 Boulevard d'Alembert, 78280 Guyancourt, France

17 Department of Physics and Astronomy “Galileo Galilei”, University of Padova, Vicolo dell'Osservatorio 3, 35122 Padova, Italy
${ }^{18}$ INAF - Astronomical Observatory of Padova, Vicolo dell'Osservatorio 5, 35122 Padova, Italy

19 CNR-IFN UOS Padova LUXOR, Via Trasea 7, 35131 Padova, Italy

20 Department of Industrial Engineering, University of Padova, Via Venezia 1, 35131 Padova, Italy

${ }^{21}$ Faculty of Engineering, University of Trento, Via Mesiano 77, 38121 Trento, Italy

${ }^{22}$ Instituto de Astrofísica de Andalucía (CSIC), c/ Glorieta de la Astronomia s/n, 18008 Granada, Spain

23 Institute of Physics and Astronomy, University of Potsdam, Potsdam, Germany

${ }^{24}$ Institut für Geophysik und extraterrestrische Physik, Technische Universität Braunschweig, Mendelssohnstr. 3, 38106 Braunschweig, Germany

25 University of Padova, Department of Physics and Astronomy "Galileo Galilei", Via Marzolo 8, 35131 Padova, Italy

${ }^{26}$ Graduate Institute of Astronomy, National Central University, 300 Chung-Da Rd, Chung-Li 32054, Taiwan

27 Space Science Institute, Macau University of Science and Technology, Avenida Wai Long, Taipa, Macau

${ }^{28}$ Department of Astronomy, University of Maryland, College Park, MD 20742-2421, USA

29 Konkoly Observatory, PO Box 67, 1525 Budapest, Hungary

${ }^{30}$ LATMOS, Sorbonne University, CNRS, UVSQ, Campus Pierre et Marie Curie, BC 102, 4 place Jussieu, 75005 Paris, France

31 DIST, Universita Parthenope, Centro Direzionale Isola C4, 80100 Napoli, Italy 
C. Tubiana et al.: Properties of dust and gas in the coma of $67 \mathrm{P}$

Appendix A: Dust and gas intensities, column densities, and production rates

Table A.1. Dust and gas measured in VIRTIS-M and OSIRIS data.

\begin{tabular}{|c|c|c|c|c|c|c|c|}
\hline \# & $A_{ \pm 45^{\circ}}$ & $A_{360^{\circ}}$ & $A f \rho(\mathrm{m})$ & $F_{ \pm 45^{\circ}}(\%)$ & $F_{ \pm 90^{\circ}, D}(\%)$ & $F_{ \pm 90^{\circ}, N}(\%)$ & $\phi\left(^{\circ}\right)$ \\
\hline $\mathrm{H}_{2} \mathrm{O}$ & $10^{-5} \mathrm{~W} \mathrm{~m}^{-2} \mathrm{sr}^{-1}$ & $10^{-5} \mathrm{~W} \mathrm{m^{-2 }} \mathrm{sr}^{-1}$ & & & & & \\
\hline 1 & $(10.0 \pm 0.2)$ & $(4.0 \pm 0.2)$ & - & 56 & 78 & 22 & 132.9 \\
\hline 2 & $(11.1 \pm 0.2)$ & $(4.1 \pm 0.2)$ & - & 60 & 83 & 17 & 108.3 \\
\hline 3 & $(12.4 \pm 0.2)$ & $(5.4 \pm 0.2)$ & - & 50 & 77 & 23 & 81.7 \\
\hline 4 & $(10.6 \pm 0.2)$ & $(5.1 \pm 0.2)$ & - & 48 & 73 & 27 & 52.8 \\
\hline 5 & $(10.5 \pm 0.2)$ & $(4.9 \pm 0.2)$ & - & 48 & 78 & 22 & 355.9 \\
\hline 6 & $(11.4 \pm 0.2)$ & $(4.8 \pm 0.2)$ & - & 51 & 77 & 27 & 311.6 \\
\hline 7 & $(11.1 \pm 0.2)$ & $(4.9 \pm 0.2)$ & - & 52 & 78 & 22 & 268.4 \\
\hline 8 & $(11.0 \pm 0.2)$ & - & - & - & - & - & 222.9 \\
\hline 9 & - & - & - & - & - & - & 216.6 \\
\hline$\overline{\mathrm{CO}_{2}}$ & $10^{-6} \mathrm{~W} \mathrm{~m}^{-2} \mathrm{sr}^{-1}$ & $10^{-6} \mathrm{~W} \mathrm{~m}^{-2} \mathrm{sr}^{-1}$ & & & & & \\
\hline 1 & $(4.8 \pm 2.0)$ & $(6.7 \pm 2.0)$ & - & 19 & 49 & 51 & 132.9 \\
\hline 2 & $(4.7 \pm 2.0)$ & $(6.6 \pm 2.0)$ & - & 19 & 47 & 53 & 108.3 \\
\hline 3 & $(4.8 \pm 2.0)$ & $(7.4 \pm 2.0)$ & - & 16 & 52 & 48 & 81.7 \\
\hline 4 & $(5.3 \pm 2.0)$ & $(7.1 \pm 2.0)$ & - & 20 & 54 & 46 & 52.8 \\
\hline 5 & $(10.6 \pm 2.0)$ & $(8.7 \pm 2.0)$ & - & 38 & 66 & 34 & 355.9 \\
\hline 6 & $(12.9 \pm 2.0)$ & $(8.9 \pm 2.0)$ & - & 38 & 52 & 48 & 311.6 \\
\hline 7 & $(10.5 \pm 2.0)$ & $(9.1 \pm 2.0)$ & - & 33 & 58 & 42 & 268.4 \\
\hline 8 & $(5.2 \pm 2.0)$ & - & - & - & - & - & 222.9 \\
\hline 9 & - & - & - & - & - & - & 216.6 \\
\hline Dust & $\left(10^{-6} \mathrm{SI}\right)$ & $\left(10^{-6} \mathrm{SI}\right)$ & & & & & \\
\hline 1 & $13.00 \pm 0.35$ & - & - & - & - & - & 132.9 \\
\hline 2 & $13.18 \pm 0.27$ & - & - & - & - & - & 108.3 \\
\hline 3 & $15.10 \pm 0.20$ & - & - & - & - & - & 81.7 \\
\hline 4 & $16.21 \pm 0.26$ & - & - & - & - & - & 52.8 \\
\hline 5 & $20.49 \pm 0.33$ & - & - & - & - & - & 355.9 \\
\hline 6 & $18.14 \pm 0.20$ & - & - & - & - & - & 311.6 \\
\hline 7 & $16.87 \pm 0.37$ & - & - & - & - & - & 268.4 \\
\hline 8 & $16.17 \pm 0.32$ & - & - & - & - & - & 222.9 \\
\hline 9 & $16.34 \pm 0.45$ & - & - & - & - & - & 216.6 \\
\hline $\bar{a}$ & $16.38 \pm 0.04$ & $8.98 \pm 0.02$ & 1.28 & 46 & 70 & 30 & 249.7 \\
\hline $\mathrm{b}$ & $15.66 \pm 0.04$ & $8.36 \pm 0.02$ & 1.19 & 47 & 68 & 32 & 220.7 \\
\hline $\mathrm{c}$ & $15.52 \pm 0.04$ & $8.39 \pm 0.02$ & 1.19 & 46 & 70 & 30 & 191.3 \\
\hline $\mathrm{d}$ & $13.46 \pm 0.04$ & $7.58 \pm 0.02$ & 1.08 & 44 & 67 & 33 & 143.6 \\
\hline $\mathrm{e}$ & $13.39 \pm 0.04$ & $7.30 \pm 0.02$ & 1.04 & 46 & 68 & 32 & 117.1 \\
\hline $\mathrm{f}$ & $15.78 \pm 0.05$ & $7.50 \pm 0.02$ & 1.07 & 53 & 73 & 27 & 88.1 \\
\hline g & $16.51 \pm 0.05$ & $7.60 \pm 0.02$ & 1.08 & 54 & 76 & 24 & 59.2 \\
\hline $\mathrm{h}$ & $16.78 \pm 0.05$ & $7.84 \pm 0.02$ & 1.12 & 53 & 77 & 23 & 44.7 \\
\hline $\mathrm{i}$ & $18.92 \pm 0.05$ & $9.22 \pm 0.02$ & 1.31 & 51 & 75 & 25 & 15.3 \\
\hline $\mathrm{j}$ & $18.95 \pm 0.05$ & $9.57 \pm 0.02$ & 1.36 & 49 & 74 & 26 & 352.1 \\
\hline $\mathrm{k}$ & $18.04 \pm 0.05$ & $8.91 \pm 0.02$ & 1.27 & 51 & 77 & 23 & 323.2 \\
\hline 1 & $18.22 \pm 0.05$ & $8.75 \pm 0.02$ & 1.25 & 52 & 75 & 25 & 294.2 \\
\hline $\mathrm{m}$ & $17.33 \pm 0.05$ & $8.62 \pm 0.02$ & 1.23 & 50 & 75 & 25 & 282.2 \\
\hline $\mathrm{n}$ & $15.22 \pm 0.05$ & $7.97 \pm 0.02$ & 1.13 & 48 & 70 & 30 & 229.1 \\
\hline
\end{tabular}

Notes. Column 1: assigned number and letter for each image; Col. 2: average flux in the $\pm 45^{\circ}$ mask (in $\mathrm{W} \mathrm{m}^{-2} \mathrm{sr}^{-1} \mathrm{~nm}^{-1}$ for the dust); Col. 3 : $\mathrm{H}_{2} \mathrm{O}$ and $\mathrm{CO}_{2}$ : average emitted band intensity calculated inside the annulus. Dust: average flux in the full annulus (in $\mathrm{W} \mathrm{m}^{-2} \mathrm{sr}^{-1} \mathrm{~nm}^{-1} \mathrm{~nm}$ ); Col. $4: A f \rho$; Cols. 5, 6, and 7: fraction of flux in the $\pm 45^{\circ}, \pm 90^{\circ}$ (subsolar) and $\pm 90^{\circ}$ (antisolar) masks, respectively, compared to the full annulus (in percent); Col. 8: subsolar longitude (in degrees). 
Table A.2. Gas column densities and production rates.

\begin{tabular}{lccccccc}
\hline \hline$\#$ & $v_{\text {out }}\left(\mathrm{m} \mathrm{s}^{-1}\right)$ & $g_{0}(\mathrm{~W} \mathrm{molec}-1)$ & $A_{360^{\circ}}\left(\mathrm{W} \mathrm{m}^{-2} \mathrm{sr}^{-1}\right)$ & $n_{360^{\circ}}\left(\mathrm{molec} \mathrm{m}^{-2}\right)$ & $Q\left(\mathrm{molec} \mathrm{s}^{-1}\right)$ & $Q\left(\mathrm{~kg} \mathrm{~s}^{-1}\right)$ & $\phi\left(^{\circ}\right)$ \\
\hline $\mathrm{H}_{2} \mathrm{O}$ & & & & & & \\
\hline 1 & 580 & $2.745 \times 10^{-23}$ & $(3.96 \pm 0.20) \times 10^{-5}$ & $(5.65 \pm 0.29) \times 10^{19}$ & $(4.06 \pm 0.21) \times 10^{26}$ & $12.16 \pm 0.61$ & 132.9 \\
2 & 580 & $2.745 \times 10^{-23}$ & $(4.14 \pm 0.20) \times 10^{-5}$ & $(5.90 \pm 0.29) \times 10^{19}$ & $(4.24 \pm 0.21) \times 10^{26}$ & $12.70 \pm 0.61$ & 108.4 \\
3 & 580 & $2.745 \times 10^{-23}$ & $(5.36 \pm 0.20) \times 10^{-5}$ & $(7.63 \pm 0.29) \times 10^{19}$ & $(5.49 \pm 0.20) \times 10^{26}$ & $16.41 \pm 0.61$ & 81.7 \\
4 & 580 & $2.745 \times 10^{-23}$ & $(5.07 \pm 0.20) \times 10^{-5}$ & $(7.22 \pm 0.29) \times 10^{19}$ & $(5.19 \pm 0.20) \times 10^{26}$ & $15.53 \pm 0.61$ & 52.8 \\
5 & 580 & $2.745 \times 10^{-23}$ & $(4.88 \pm 0.20) \times 10^{-5}$ & $(6.95 \pm 0.29) \times 10^{19}$ & $(5.00 \pm 0.20) \times 10^{26}$ & $14.95 \pm 0.61$ & 355.9 \\
6 & 580 & $2.745 \times 10^{-23}$ & $(4.77 \pm 0.20) \times 10^{-5}$ & $(6.78 \pm 0.29) \times 10^{19}$ & $(4.88 \pm 0.20) \times 10^{26}$ & $14.60 \pm 0.61$ & 311.6 \\
7 & 580 & $2.745 \times 10^{-23}$ & $(4.91 \pm 0.20) \times 10^{-5}$ & $(6.99 \pm 0.29) \times 10^{19}$ & $(5.03 \pm 0.20) \times 10^{26}$ & $15.03 \pm 0.61$ & 268.4 \\
\hline $\mathrm{CO}_{2}$ & & & & & & & \\
\hline 1 & 380 & $1.25 \times 10^{-22}$ & $(6.7 \pm 2.0) \times 10^{-6}$ & $(2.11 \pm 0.63) \times 10^{18}$ & $(0.99 \pm 0.30) \times 10^{25}$ & $0.73 \pm 0.22$ & 132.9 \\
2 & 380 & $1.25 \times 10^{-22}$ & $(6.6 \pm 2.0) \times 10^{-6}$ & $(2.08 \pm 0.63) \times 10^{18}$ & $(0.98 \pm 0.30) \times 10^{25}$ & $0.72 \pm 0.22$ & 108.3 \\
3 & 380 & $1.25 \times 10^{-22}$ & $(7.4 \pm 2.0) \times 10^{-6}$ & $(2.33 \pm 0.63) \times 10^{18}$ & $(1.10 \pm 0.29) \times 10^{25}$ & $0.80 \pm 0.22$ & 81.7 \\
4 & 380 & $1.25 \times 10^{-22}$ & $(7.1 \pm 2.0) \times 10^{-6}$ & $(2.23 \pm 0.63) \times 10^{18}$ & $(1.05 \pm 0.29) \times 10^{25}$ & $0.77 \pm 0.22$ & 52.8 \\
5 & 380 & $1.25 \times 10^{-22}$ & $(8.7 \pm 2.0) \times 10^{-6}$ & $(2.71 \pm 0.63) \times 10^{18}$ & $(1.28 \pm 0.29) \times 10^{25}$ & $0.93 \pm 0.22$ & 355.9 \\
6 & 380 & $1.25 \times 10^{-22}$ & $(8.9 \pm 2.0) \times 10^{-6}$ & $(2.78 \pm 0.63) \times 10^{18}$ & $(1.31 \pm 0.29) \times 10^{25}$ & $0.96 \pm 0.22$ & 311.6 \\
7 & 380 & $1.25 \times 10^{-22}$ & $(9.1 \pm 2.0) \times 10^{-6}$ & $(2.84 \pm 0.63) \times 10^{18}$ & $(1.34 \pm 0.29) \times 10^{25}$ & $0.98 \pm 0.22$ & 268.4 \\
\hline
\end{tabular}

Notes. Column 1: assigned number for each image; Col. 2: gas outflow speed (Fink et al. 2016); Col. 3: $g$ factor for the $\mathrm{H}_{2} \mathrm{O}$ and $\mathrm{CO}_{2}$ bands at 1 $\mathrm{au}$; Col. 4: average emitted band intensity calculated inside the annulus; Col. 5: average column density calculated inside the annulus; Cols. 6 and 7: $\mathrm{H}_{2} \mathrm{O}$ and $\mathrm{CO}_{2}$ production rate in molec. $\mathrm{s}^{-1}$ and $\mathrm{kg} \mathrm{s}^{-1}$, respectively; Col. 8: subsolar longitude. 